\title{
High burden of cerebral white matter lesion in 9 Asian cities
}

\author{
Bonnie Yin Ka Lam ${ }^{1,2,3}$, Brian Yiv ${ }^{1,2}$, Encarnita Ampil ${ }^{4,5}$, Christopher Li-Hsian Chen ${ }^{6,7}$, \\ Yustiani Dikot ${ }^{8}$, Jacqueline C. Dominguez ${ }^{5}$, Patel Vishal Ganeshbhai ${ }^{9}$, Saima Hilal ${ }^{6,7,10}$, \\ Nagaendran Kandiah ${ }^{11}$, SangYun Kim ${ }^{12}$, Jun-Young Lee ${ }^{13}$, Anam Paulus Ong ${ }^{14}$, \\ Vorapun Senanarong ${ }^{15}$, Kam Tat Leung ${ }^{1,2}$, Huali Wang ${ }^{16}$, Yuan-Han Yang ${ }^{17}$, Tingting Yong ${ }^{11}$, \\ Faheem Arshad $^{9}$, Suvarna Alladi ${ }^{9}$, Samuel Wong ${ }^{18}$, Ho Ko $^{1,2,19}$, AlexanderYuk Lun Lau ${ }^{1,2 \bowtie ~ \& ~}$ \\ Vincent Chung Tong Mok ${ }^{1,2,3}$
}

Age-related white matter lesion (WML) is considered a manifestation of sporadic cerebral small vessel disease and an important pathological substrate for dementia. Asia is notable for its large population with a looming dementia epidemic. Yet, the burden of WML and its associated risk factors across different Asian societies are unknown. Subjects from 9 Asian cities (Bangkok, Bandung, Beijing, Bengaluru, Hong Kong, Kaohsiung, Manila, Seoul, and Singapore) were recruited $(n=5701)$ and classified into (i) stroke/transient ischemic attack (TIA), (ii) Alzheimer's disease (AD)/mild cognitive impairment $(\mathrm{MCl})$, or (iii) control groups. Data on vascular risk factors and cognitive performance were collected. The severity of WML was visually rated on MRI or CT. The prevalence of moderateto-severe WML was the highest in subjects with stroke/TIA (43.3\%). Bandung Indonesia showed the highest prevalence of WML, adjusted for age, sex, education, disease groups, and imaging modality. Hypertension and hyperlipidemia were significant risk factors for WML, and WML was negatively associated with MMSE in all groups. WML is highly prevalent in Asia and is associated with increasing age, hypertension, hyperlipidemia, and worse cognitive performance. Concerted efforts to prevent WML will alleviate the huge dementia burden in the rapidly aging Asian societies.

Asia had the largest number of people suffering from dementia (22.9 million), which was more than twice the numbers in Europe (10.5 million) or the Americas ( 9.4 million), as recorded in the global impact of dementia in the World Alzheimer Report $2015^{1}$. This number was estimated to triple to 67 million in $2050-2$ to 3 times higher than the estimates for Europe (19 million) or the Americas (30 million) ${ }^{1}$. Devising and implementing preventive strategies against dementia are of paramount importance, particularly in Asia.

Subclinical sporadic cerebral small vessel disease (CSVD) has been increasingly recognized in recent years to be a prevalent and important pathological substrate for cognitive impairment and dementia ${ }^{2}$. Subclinical sporadic

${ }^{1}$ Division of Neurology, Department of Medicine and Therapeutics, The Chinese University of Hong Kong, Hong Kong SAR, China. ${ }^{2}$ Therese Pei Fong Chow Research Centre for Prevention of Dementia and Margaret Kam Ling Cheung Research Centre for Management of Parkinsonism, Gerald Choa Neuroscience Centre, The Chinese University of Hong Kong, Hong Kong SAR, China. ${ }^{3}$ Shenzhen Research Institute, The Chinese University of Hong Kong, Shenzhen, China. ${ }^{4}$ Department of Neuroscience and Behavioural Medicine, Faculty of Medicine and Surgery, University of Santo Tomas, Manila, Philippines. ${ }^{5}$ Institute for Neurosciences, St. Luke's Medical Centre, Quezon City, Philippines. ${ }^{6}$ Memory Aging and Cognition Center, National University Health System, Singapore, Singapore. ${ }^{7}$ Department of Pharmacology, National University of Singapore, Singapore, Singapore. ${ }^{8}$ Department of Neurology, Achmad Yani University, Cimahi, Indonesia. ${ }^{9}$ Department of Neurology, National Institute of Mental Health and Neuro Sciences, Bengaluru, India. ${ }^{10}$ Saw Swee Hock School of Public Health, National University of Singapore and National University Health System, Singapore, Singapore. ${ }^{11}$ Department of Neurology, National Neuroscience Institute, Singapore, Singapore. ${ }^{12}$ Department of Neurology, Seoul National University College of Medicine, Seoul National University Bundang Hospital, Seoul, South Korea. ${ }^{13}$ Department of Psychiatry, SMG-SNU Boramae Medical Centre, Seoul National University College of Medicine, Seoul, South Korea. ${ }^{14}$ Department of Neurology, Hasan Sadikin Hospital, Bandung, Indonesia. ${ }^{15}$ Department of Medicine At, Mahidol University, Bangkok, Thailand. ${ }^{16}$ Dementia Care and Research Centre, Peking University Institute of Mental Health, Beijing, China. ${ }^{17}$ Department of Neurology, Kaohsiung Municipal Ta-Tung Hospital and Kaohsiung Medical University Hospital, Kaohsiung Medical University, Kaohsiung, Taiwan. ${ }^{18} \mathrm{JC}$ School of Public Health and Primary Care, The Chinese University of Hong Kong, Hong Kong SAR, China. ${ }^{19} \mathrm{Li}$ Ka Shing Institute of Health Sciences, Faculty of Medicine, The Chinese University of Hong Kong, Hong Kong SAR, China. ${ }^{\circledR}$ email: alexlau@cuhk.edu.hk 
CSVD commonly manifests on neuroimaging as white matter lesion (WML), lacunes, microbleeds, enlarged perivascular space, or microinfarcts ${ }^{3,4}$. In patients with stroke, variable severity levels of cognitive impairment are present in more than $80 \%$ of patients ${ }^{5}$ and the presence of subclinical CSVD lesion is associated with worse cognitive performance ${ }^{6-12}$. Its presence significantly increases the risk of poststroke dementia ${ }^{9,13}$. Among those who survive stroke without early-onset poststroke dementia, the presence of CSVD can also increase the risk of delayed-onset poststroke dementia ${ }^{9}$.

In patients with Alzheimer's disease (AD), CSVD is associated with worse cognitive performance and more rapid cognitive decline. Among those with mild cognitive impairment (MCI), it also increases the risk of conversion to $\mathrm{AD}^{14,15}$. In addition, some studies have even suggested a causative role of CSVD in AD pathologies ${ }^{16,17}$. Overall, population studies showed that the presence of CSVD increases the risk of incident vascular dementia or $\mathrm{AD}^{18,19}$.

Apart from dementia, subclinical CSVD also increases the risk of incident stroke ${ }^{19}$. Other clinical manifestations of CSVD may include depression and other behavioral problems (e.g. apathy), gait and postural instability, and urinary incontinence, which may occur in isolation or in conjunction with dementia/post-stroke syndrome. Overall, CSVD increases the risk of functional decline and mortality ${ }^{19,20}$.

While many risk factors that may be associated with sporadic CSVD, age and hypertension are the most consistent factors $^{21}$. In addition, cerebral WML was found to be highly heritable and recent studies have identified an increasing number of genetic loci associated with CSVD $^{22}$. Given the multi-factorial nature of sporadic CSVD, the burden of CSVD may vary across geographical locations and cultures. The first population-based studies comparing the prevalence of sporadic subclinical CSVD between Asians (Chinese) and Australians suggested that Asians may have a higher prevalence of CSVD than Australians, even after adjusting for vascular risk factors ${ }^{23}$. Although few studies had reported the prevalence of subclinical CSVD in stroke patients in different Asia cities, comparison between studies of different cities was difficult because the studies used different scales for the quantification of CSVD ${ }^{24-26}$. Moreover, very few studies reported the CSVD prevalence in AD in Asia $^{11}$. There was limited data on CSVD in some cities such as Indonesia, the Philippines, and Thailand. Finally, no studies had compared the prevalence of subclinical CSVD in stroke and AD subjects in different age groups using a standardized CSVD rating method.

The AWARE (Asian White mAtteR lEsion) study group initiated a joint international effort across multiple Asian centers to estimate the burden of CSVD (using WML as a surrogate marker for CSVD) in Asia. The study group reported the largest community (stroke- and dementia-free subjects) study ( $n=1797)$ on the prevalence of subclinical CSVD previously in Asia, which again showed a high prevalence of CSVD in Asia ${ }^{27}$. Apart from community subjects, the AWARE study group also collected data from subjects with stroke/TIA $(n=1834)$ and $\mathrm{AD} / \mathrm{MCI}(n=2070)$. The objective of the present study was to estimate the burden of cerebral WML in stroke/ TIA and AD/MCI across 9 cities in Asia. We also included community control groups from the AWARE to compare against the results of stroke/TIA and AD/MCI groups. We also investigated the risk factors and cognitive impact of WML among all subjects and different clinical groups. The hypotheses were: (i) the overall prevalence of WML in Asia is high but there could be variations in the different Asian cities; (ii) vascular risk factor such as hypertension may be a common contributor to the WML and WML is associated with worse cognitive function across the Asian regions.

\section{Methods}

Subjects and recruitment procedure. The AWARE study included a total of 5,701 subjects. Subjects consisted of patients with stroke/TIA $(n=1834)$, AD/MCI $(n=2070)$, and controls $(n=1797)$ across 9 Eastern and South-Eastern Asian cities (Bangkok, Bandung, Beijing, Bengaluru, Hong Kong, Kaohsiung, Manila, Seoul, Singapore). Patients with AD dementia or MCI were assessed and recruited in cognitive disorder clinics. Patients were diagnosed with $\mathrm{AD}$ dementia according to the NINCDS-ADRDA criteria ${ }^{28}$. Patients were classified as having MCI if there were (i) subjective cognitive complaints; (ii) objective cognitive impairment fulfilled that of local standards in defining cognitive impairment, and (iii) presenting with no or minimal functional impairment in daily functioning. Patients with mixed dementia cases were excluded $(n=197)$, and hence, none of the patients with $\mathrm{AD}$ dementia or MCI had a history of overt stroke. We included both hemorrhagic and ischemic stroke in the stroke group. We defined stroke according to clinical evidence of cerebral injury based on symptoms persisting $24 \mathrm{~h}$ or longer, and other etiologies excluded. We defined TIA based on transient neurological deficits (less than $24 \mathrm{~h}$ ) and the absence of infarcts/hemorrhage on neuroimaging ${ }^{29}$. Based on available data from each cohort, we defined dementia in stroke/TIA patients if they had a clinical dementia rating scale of 1 or above, a mini-mental state examination (MMSE) of 16 or below ${ }^{30}$ or they were diagnosed by respective dementia experts to have vascular dementia according to the NINDS-AIREN criteria ${ }^{31}$. Healthy controls were defined as community-dwelling subjects without history of known cognitive disorder and/or MMSE scored less than education-adjusted cutoff for dementia; without other significant neurological/psychiatric comorbidities, e.g. stroke, multiple sclerosis, psychosis; or severe medical illnesses and were functionally independent ${ }^{27}$.

Ethics approval of each cohort was obtained from the review board of the affiliated university. Ethics approval of the Bangkok cohort was obtained from the Central Institutional Review Board of Mahidol University; the Bandung cohort was obtained from Dr. Hasan Sadikin General Hospital and the Faculty of Medicine, Universitas Padjadjaran Health Research Ethics Committee; the Beijing cohort was obtained from the institutional review board of Peking University Institute of Mental Health (Sixth Hospital); the Bengaluru cohort was obtained from the Nizam's Institute of Medical Sciences ethics committee; the Hong Kong cohort was obtained from the Joint Chinese University of Hong Kong-New Territories East Cluster Clinical Research Ethics Committee; the Kaohsiung cohort was obtained from the institutional review board of the Kaohsiung Medical University Hospital; the Manila cohort was obtained from the St. Luke's Medical Center Institutional Ethics Review Committee; the 
Seoul cohort was obtained from the Institutional Review Board of Seoul National University Bundang Hospital while the Singapore cohort was obtained from the Singapore Eye Research Institute and National Healthcare Group Domain-Specific Review Board.

Clinical and cognitive measures. Clinical and cognitive measures such as age, sex, education years, and MMSE were collected. Patients were defined as having hypertension if systolic blood pressure $\geq 140 \mathrm{~mm} \mathrm{Hg}$ and/ or diastolic blood pressure $\geq 90 \mathrm{~mm} \mathrm{Hg}$, had a history of hypertension, or were using antihypertensive medication. Diabetes mellitus (DM) was defined as a fasting serum glucose level of $7.0 \mathrm{mmol} / \mathrm{L}$ or higher, a postprandial serum glucose level of $11.1 \mathrm{mmol} / \mathrm{L}$ or higher, or the use of oral hypoglycemic agents/insulin. Hyperlipidemia was defined as a total cholesterol level of $5.2 \mathrm{mmol} / \mathrm{L}$ or higher, a low-density lipoprotein cholesterol level of $2.6 \mathrm{mmol} / \mathrm{L}$ or higher, a triglyceride level of $1.70 \mathrm{mmol} / \mathrm{L}$ or higher, or the use of lipid-lowering drugs ${ }^{32}$.

Imaging. Subjects with either magnetic resonance imaging (MRI) or computerized tomography (CT) were eligible for this study. For details of the scanner information, please see Supplementary Table S1a,b, and Supplementary Fig. 1. The severity of WMLs was rated on axial MRI FLAIR or CT scan. If both MRI and CT were available, rating was performed on MRI (see Supplementary Table S2).To assess inter-rater agreement between different raters, Hong Kong acted as the central rater, and each center provided 30 raw images to Hong Kong for evaluation of the inter-rater agreement.

Measurement of WML. The severity of WML for each subject was rated according to the modified Fazekas scale $^{33}$ or the Age-Related White Matter Changes Scale (ARWMC) ${ }^{34}$ based on the MRI axial FLAIR sequence or CT. We used the operationalized global Fazekas rating, which has a score ranging from 0 to $3^{35}$. We also used the operationalized global ARWMC score, with a score ranging from 0 to $3^{34}$. We defined the presence of moderate-to-severe WML by a global score of $\geq 2$ for both Fazekas and ARWMC scale. The Hong Kong community dataset with both Fazekas and ARWMC global ratings showed that the correlation between the scales was high $(p<0.0001 ; r=0.943)$. Both visual rating scales of WML showed good agreement between CT and MRI ${ }^{36}$.

Statistical analysis. Data in continuous variables were first examined by a test of normality. Group comparisons with clinical variables and demographic information were performed using the Kruskal-Wallis test for continuous variables while the chi-square test was used for categorical variables $(p<0.05)$. Post-hoc analyses between cities were conducted. Multiple logistic regression models were used to study the association between vascular risk factors and prevalence of moderate-to-severe WML with adjustment for age, sex and level of education, Asian regions, disease groups, and imaging modality as appropriate. Multiple linear regression models were used to study the association between the prevalence of moderate-to-severe WML and MMSE with adjustment for age, sex, and level of education, Asian regions, and hypertension. In addition, we stratified the subjects into age groups among disease groups $(\leq 60,61-70,71-80$, and $\geq 81$ years old) to compare the associations between vascular risk factors and prevalence of moderate-to-severe WML. Pairwise deletion was applied on missing data. Bonferroni correction was used for multiple comparison correction. All analyses were performed using IBM SPSS (IBM SPSS Statistics for Mac OS, Version 24.0).

\section{Results}

The CONSORT diagram shows the study inclusions, as well as the data available from each center (see Fig. 1). Table 1 shows the clinical demographics of the 3 groups: (i) stroke/TIA group; (ii) AD/MCI group, and (iii) control group. The prevalence of moderate-to-severe WML (defined as $\geq 2$ on visual rating) in stroke/TIA, AD/ MCI and control groups were $43.3 \%, 38.2 \%$ and $36.7 \%$ respectively (Table 1 ).

Agreement in WML rating standard was first established before data analysis. The Fazekas scale was used as the tool for inter-rater measurement as most countries adopted this scale. The intra-class correlation (ICC) range from fair to excellent in different centers $(0.59-0.78)$, for the ICC results between each center and Hong Kong, please refer to supplementary results.

Prevalence of WML with age. The prevalence of moderate-to-severe WML in different age groups was examined. Age was divided into four groups and the overall prevalence of moderate-to-severe WML increased with age. The positive trend between age and the prevalence of moderate-to-severe WML was consistent in all groups (Fig. 2). The prevalence of the stroke/TIA group was $32.2 \%$ at $\leq 60$ years old but increased to $64.2 \%$ at $\geq 81$ years old. Prevalence of moderate-to-severe WML in the AD/MCI group was $20.0 \%$ at $\leq 60$ years old and increased at a relatively moderate rate to $49.1 \%$ at $\geq 81$ years old (Fig. 2). The control group had a relatively lower WML at a younger age $(8.3 \%$ at $\leq 60$ years old) but its prevalence increased proportionally with age and its prevalence reached $65.2 \%$, matching that in the stroke/TIA group at $\geq 81$ years old. The age-stratified risk for having moderate-to-severe WML increases with age and was highest in the control group (see Supplementary Table S3).

Prevalence of WML in specific disease groups and controls. Clinical demographics of various Asian cohorts in specific disease groups and controls were presented in Tables 2, 3 and 4.

Hong Kong, Kaohsiung, Bandung, Bangkok, Singapore, and Bengaluru contributed data to the stroke/TIA group. When assessing the prevalence of moderate-to-severe WML using the $\geq 2$-cutoff, Bandung showed the highest prevalence of moderate-to-severe WML (88.9\%) while the Kaohsiung had the lowest prevalence of $30.0 \%$ (see Table 2). Data from Bengaluru $(n=27)$ had a relatively small sample size and were excluded from the 


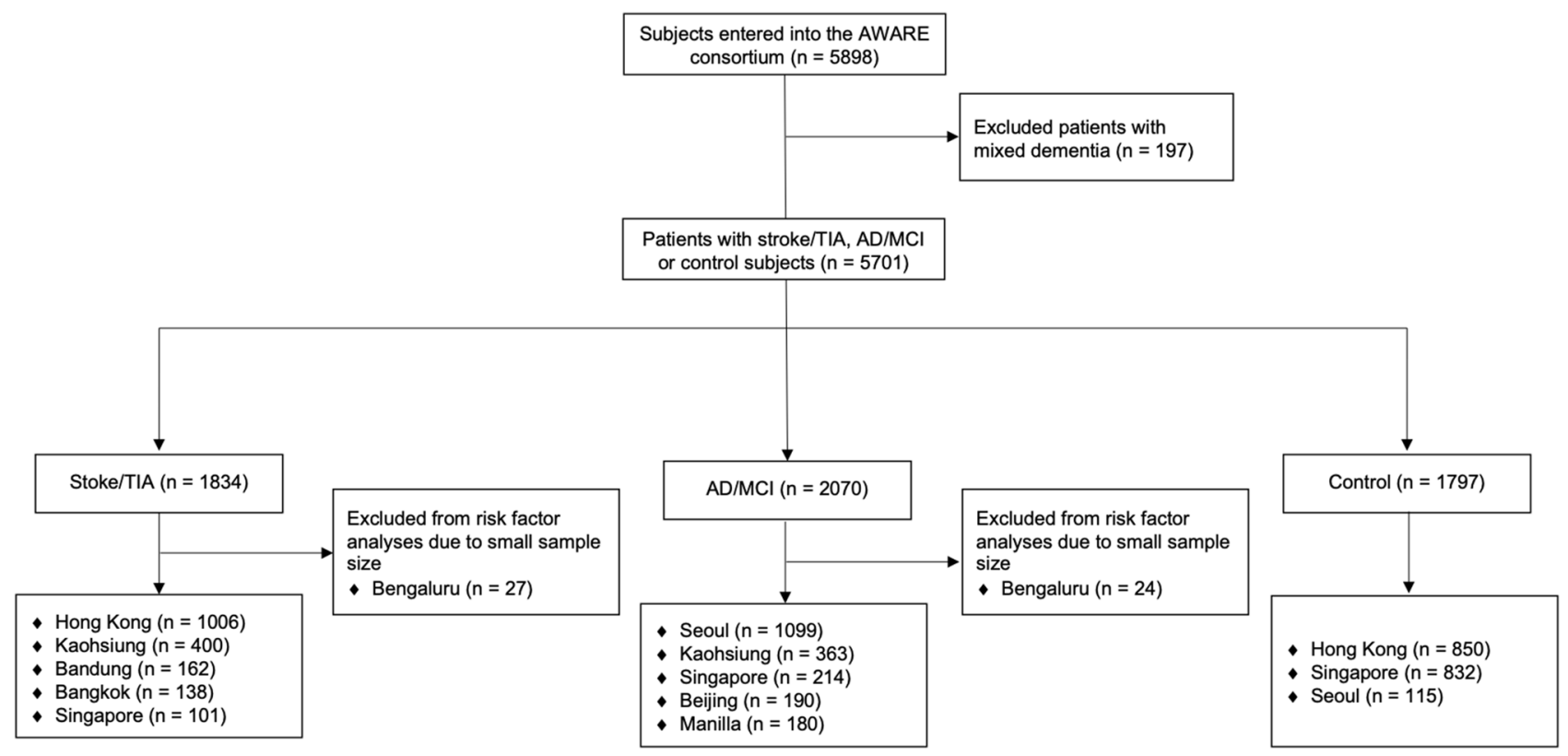

Figure 1. CONSORT diagram of the AWARE study.

\begin{tabular}{|c|c|c|c|c|}
\hline & Stroke/TIA $(n=1834)$ & AD/MCI $(n=2070)$ & Controls $(n=1797)$ & $p$ \\
\hline Age, $y r$, mean \pm SD $(\min ; \max )$ & $67.73 \pm 12.16(15 ; 98)$ & $76.30 \pm 8.67(37 ; 99)$ & $70.29 \pm 6.00(60 ; 89)$ & $p<0.001^{\mathrm{a}, \mathrm{b}, \mathrm{c}}$ \\
\hline Education, yr, mean \pm SD (min; max) & $6.35 \pm 5.02(0 ; 30)$ & $8.28 \pm 5.57(0 ; 22)$ & $7.19 \pm 4.79(0 ; 22)$ & $p<0.001^{\mathrm{a}, \mathrm{b}, \mathrm{c}}$ \\
\hline Female; $n(\%)$ & $806(43.9 \%)$ & $1319(63.7 \%)$ & $1024(57.0 \%)$ & $p<0.001^{\mathrm{a}, \mathrm{b}, \mathrm{c}}$ \\
\hline Hypertension; $n(\%)^{\mathrm{i}}$ & $1315(71.9 \%)$ & $1079(53.2 \%)$ & $1220(67.9 \%)$ & $p<0.001^{\mathrm{a}, \mathrm{b}, \mathrm{c}}$ \\
\hline Diabetes mellitus; $n(\%)^{\mathrm{ii}}$ & $665(36.4 \%)$ & $527(26.1 \%)$ & $529(29.5 \%)$ & $p<0.001^{\mathrm{a}, \mathrm{b}, \mathrm{c}}$ \\
\hline Hyperlipidemia; $\mathrm{n}(\%)^{\mathrm{iii}}$ & $1016(55.8 \%)$ & $470(28.2 \%)$ & $909(50.6 \%)$ & $p<0.001^{\mathrm{a}, \mathrm{b}, \mathrm{c}}$ \\
\hline Ischemic heart disease; $n(\%)^{\mathrm{iv}}$ & $198(11.4 \%)$ & $205(11.7 \%)$ & 1 & $p=0.837$ \\
\hline Atrial fibrillation; $n(\%)^{\mathrm{v}}$ & $258(14.1 \%)$ & $42(2.7 \%)$ & 1 & $p<0.001$ \\
\hline Smoker (either current or past); $n(\%)^{\text {vi }}$ & $615(34.3 \%)$ & $159(17.9 \%)$ & $419(23.3 \%)$ & $p<0.001^{\mathrm{a}, \mathrm{b}, \mathrm{c}}$ \\
\hline MMSE, mean \pm SD $(\min ; \max )$ & $22.04 \pm 6.98(0 ; 30)$ & $18.90 \pm 6.12(0 ; 30)$ & $25.6 \pm 3.48(2 ; 30)$ & $p<0.001^{\mathrm{a}, \mathrm{b}, \mathrm{c}}$ \\
\hline $\mathrm{WML}=0$ & $499(27.2 \%)$ & $269(13.0 \%)$ & $258(14.4 \%)$ & $p<0.001^{\mathrm{a}, \mathrm{b}, \mathrm{c}}$ \\
\hline $\mathrm{WML}=1$ & $541(29.5 \%)$ & $1011(48.8 \%)$ & $880(49.0 \%)$ & \\
\hline $\mathrm{WML}=2$ & $416(22.7 \%)$ & $495(23.9 \%)$ & $533(29.7 \%)$ & \\
\hline $\mathrm{WML}=3$ & $378(20.6 \%)$ & $295(14.3 \%)$ & $126(7.0 \%)$ & \\
\hline Prevalence of moderate to severe WML $(\geq 2) ; n(\%)$ & $794(43.3 \%)$ & $790(38.2 \%)$ & $659(36.7 \%)$ & $p<0.001^{\mathrm{a}, \mathrm{c}}$ \\
\hline
\end{tabular}

Table 1. Clinical demographics of different disease groups and controls in the AWARE study. This table illustrated the clinical demographics of the disease groups and controls in the AWARE study. For post-hoc group comparisons, a $\mathrm{AD} / \mathrm{MCI}$ versus stroke/TIA. ${ }^{\mathrm{b}} \mathrm{AD} / \mathrm{MCI}$ versus controls. ${ }^{\mathrm{c} S t r o k e / T I A}$ versus controls. Total number of inclusion cases were ${ }^{\mathrm{i}} n=5653 ;{ }^{\mathrm{ii}} n=5643 ;{ }^{\mathrm{iii}} n=5286 ;{ }^{\mathrm{iv}} n=3497 ;{ }^{\mathrm{v}} n=3366 ;{ }^{\mathrm{vi}} n=4478$. MCI Mild cognitive impairment, $A D$ Alzheimer's disease, TIA Transient ischaemic attack, MMSE Mini-mental state examination.

ANOVA and the subsequent regression analyses. Brief information about the Bengaluru group was specified in the supplementary results.

Seoul, Kaohsiung, Singapore, Beijing, Manila, and Bengaluru contributed data to the AD/MCI group. Among this group, both Kaohsiung and Singapore showed a very high prevalence of WML with gradings 2 or above (58.4\% and $57.9 \%$ respectively) while Manilla showed the lowest (18.9\%) (see Table 3). Data from Bengaluru had a relatively small sample size $(n=24)$ and were excluded from the ANOVA analysis, as well as the subsequent regression analyses. Brief information about the Bengaluru group was included in the supplementary results.

The control group consists of data from Hong Kong, Singapore, and Seoul. Singapore showed the highest prevalence of moderate-to-severe WML (47.5\%), followed by Hong Kong (28.8\%) while Seoul showed the lowest (16.5\%) (see Table 4). 


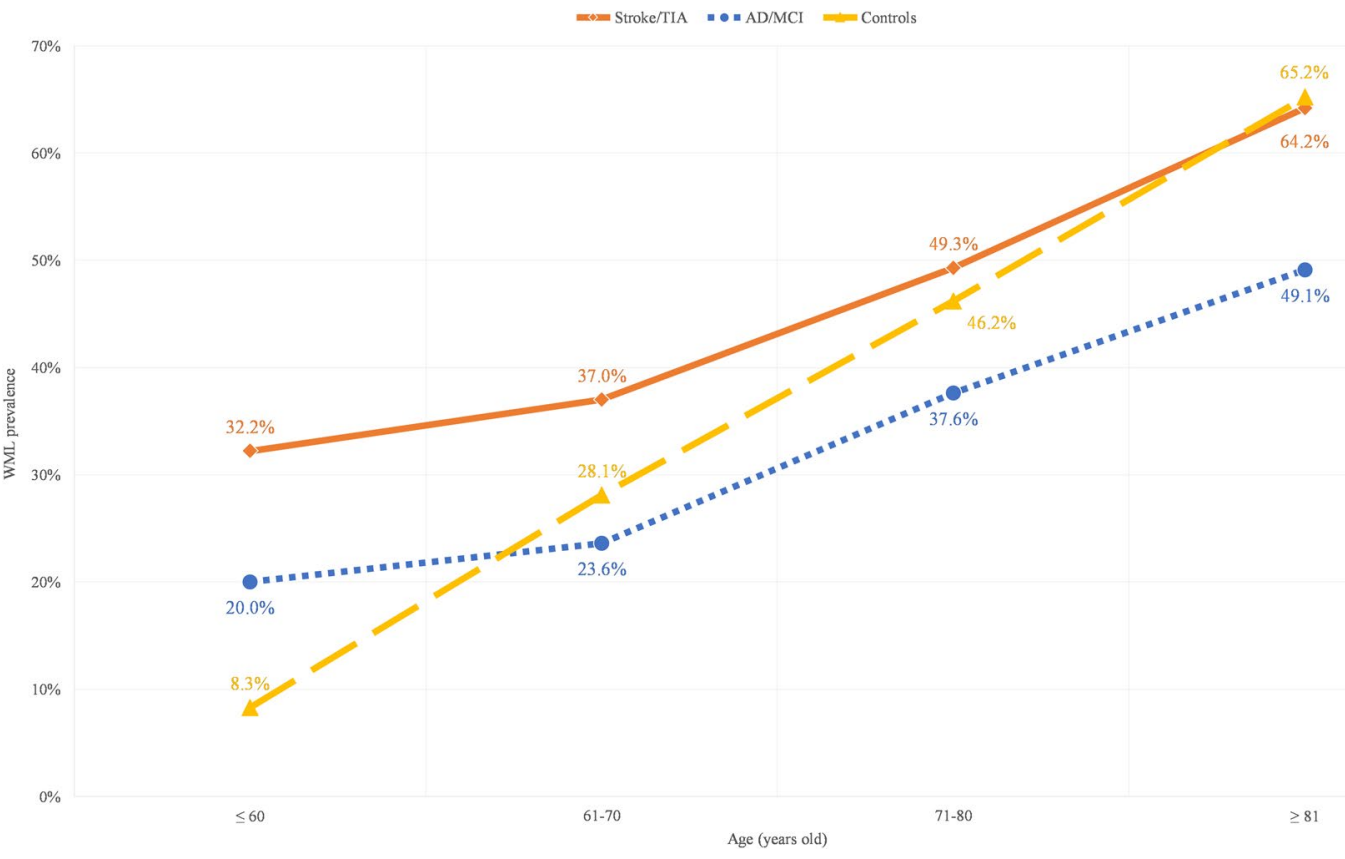

Figure 2. The prevalence of severe white matter lesion in different age groups in the AD/MCI group, stroke/ TIA group, and control group.

\begin{tabular}{|c|c|c|c|c|c|c|}
\hline & Hong Kong $(n=1006)$ & Kaohsiung $(n=400)$ & Bandung $(n=162)$ & Bangkok $(n=138)$ & Singapore $(n=101)$ & $p^{*}$ \\
\hline Age, year, mean \pm SD (min; max $)$ & $69.6 \pm 11.67(20 ; 98)$ & $68.49 \pm 12.17(34 ; 94)$ & $58.69 \pm 11.08(31 ; 84)$ & $62.21 \pm 13.55(15 ; 91)$ & $68.63 \pm 7.18(52 ; 92)$ & $p<0.001^{\mathrm{a}, \mathrm{b}, \mathrm{f}, \mathrm{g}, \mathrm{h}, \mathrm{i}}$ \\
\hline $\begin{array}{l}\text { Education, year, mean } \pm \text { SD (min; } \\
\max \text { ) }\end{array}$ & $5.57 \pm 4.69(0 ; 30)$ & $6.43 \pm 5.19(0 ; 20)$ & $6.53 \pm 4.14(0 ; 18)$ & $9.63 \pm 5.78(0 ; 18)$ & $8.62 \pm 4.98(0 ; 22)$ & $p<0.001^{\mathrm{a}, \mathrm{c}, \mathrm{d}, \mathrm{eg}, \mathrm{gh}, \mathrm{j}}$ \\
\hline Female; $n(\%)$ & $445(44.2 \%)$ & $157(39.3 \%)$ & $90(55.6 \%)$ & $55(39.9 \%)$ & $53(52.5 \%)$ & $p=0.003^{\mathrm{i}}$ \\
\hline Hypertension; $n(\%)$ & $698(69.4 \%)$ & $305(76.3 \%)$ & $132(81.5 \%)$ & $88(64.2 \%)$ & $73(72.3 \%)$ & $p=0.001^{\mathrm{b}, \mathrm{e}}$ \\
\hline Diabetes mellitus; $n$ (\%) & $364(36.2 \%)$ & $176(44.0 \%)$ & $41(25.3 \%)$ & $46(33.6 \%)$ & $30(29.7 \%)$ & $p<0.001^{\mathrm{i}}$ \\
\hline Hyperlipidemia; $n(\%)$ & $610(60.6 \%)$ & $200(50.0 \%)$ & $68(42.0 \%)$ & $56(41.2 \%)$ & $74(73.3 \%)$ & $p<0.001^{\mathrm{a}, \mathrm{b}, \mathrm{d}, \mathrm{f}, \mathrm{h}, \mathrm{j}}$ \\
\hline Ischemic heart disease; $n(\%)$ & $93(9.2 \%)$ & $45(11.3 \%)$ & $30(18.5 \%)$ & $12(22.2 \%)$ & $17(16.8 \%)$ & $p<0.001^{\mathrm{a}, \mathrm{b}}$ \\
\hline Atrial fibrillation; $n(\%)$ & $159(15.8 \%)$ & $62(15.5 \%)$ & $27(16.7 \%)$ & $0(0.0 \%)$ & $8(7.9 \%)$ & $p<0.001^{\text {a,ef,g }}$ \\
\hline Smoker (either current or past); $n(\%)$ & $397(40.0 \%)$ & $119(29.8 \%)$ & $50(30.9 \%)$ & $37(26.8 \%)$ & $12(11.9 \%)$ & $p<0.001^{\mathrm{a}, \mathrm{a}, \mathrm{d}, \mathrm{f}, \mathrm{h}, \mathrm{j}}$ \\
\hline MMSE, mean \pm SD (min; max $)$ & $24.05 \pm 5.86(4 ; 30)$ & $15.24 \pm 6.45(0 ; 28)$ & $22.93 \pm 5.21(7 ; 30)$ & $24.35 \pm 6.38(5 ; 30)$ & $24.56 \pm 5.43(10 ; 30)$ & $p<0.001^{\mathrm{d}, \mathrm{g}, \mathrm{i}, \mathrm{j}}$ \\
\hline $\mathrm{WML}=0$ & $396(39.4 \%)$ & $30(7.5 \%)$ & $11(6.8 \%)$ & $60(43.5 \%)$ & $2(2.0 \%)$ & $p<0.001^{\text {a,b,c,d,e,f,g,h,i }}$ \\
\hline $\mathrm{WML}=1$ & $203(20.2 \%)$ & $250(62.5 \%)$ & $7(4.3 \%)$ & $21(15.2 \%)$ & $56(55.4 \%)$ & \\
\hline $\mathrm{WML}=2$ & $203(20.3 \%)$ & $86(21.5 \%)$ & $29(17.9 \%)$ & $57(41.3 \%)$ & $34(33.7 \%)$ & \\
\hline $\mathrm{WML}=3$ & $204(20.3 \%)$ & $34(8.5 \%)$ & $115(71.0 \%)$ & $0(0.0 \%)$ & $9(8.9 \%)$ & \\
\hline $\begin{array}{l}\text { Prevalence of moderate to severe } \\
\text { WML }(\geq 2) ; n(\%)\end{array}$ & $407(40.5 \%)$ & $120(30.0 \%)$ & $144(88.9 \%)$ & $57(41.3 \%)$ & $43(42.6 \%)$ & $p<0.001^{\mathrm{b}, \mathrm{d}, \mathrm{eh}, \mathrm{i}}$ \\
\hline
\end{tabular}

Table 2. Clinical demographics of the stroke/TIA group. This table showed the clinical demographics of the stroke/TIA group. ${ }^{a}$ Hong Kong versus Bangkok. ${ }^{b}$ Hong Kong versus Bandung. ${ }^{c}$ Hong Kong versus Singapore. ${ }^{\mathrm{d}}$ Hong Kong versus Kaohsiung. ${ }^{\mathrm{e}}$ Bangkok versus Bandung. ${ }^{\mathrm{f}}$ Bangkok versus Singapore. ${ }^{\text {g Bangkok versus }}$ Kaohsiung. handung versus Singapore. ${ }^{\mathrm{i}}$ Bandung versus Kaohsiung. ${ }^{j}$ Singapore versus Kaohsiung. MMSE Mini-mental state examination, WML White matter lesion. ${ }^{\star}$ Bonferroni correction is applied to the alpha $(\alpha=0.005)$.

Risk factors of moderate-to-severe white matter lesion. Multiple logistic regression was performed particularly to ascertain the effects of hypertension, hyperlipidemia, and diabetes mellitus on moderate-tosevere WML on all subjects. Age, sex, level of education, Asian cities, disease groups, and imaging modality were entered as covariates as appropriate. Predictors were only regarded as significant if $p \leq 0.0125$, corrected for multiple comparisons using Bonferroni correction.

The regression model showed the significant risk factors for moderate-to-severe WML in all subjects were hypertension $(p<0.001 ; \mathrm{OR}=1.81,95 \% \mathrm{CI}=1.58-2.08)$ and hyperlipidemia $(p=0.012 ; \mathrm{OR}=1.19,95 \%$ $\mathrm{CI}=1.04-1.36$ ) (Table 5). Hypertension was also a significant predictor for moderate-to-severe WML, after adjustment for age, sex, education and Asian cities and image modality in the stroke/TIA $(p<0.001 ; \mathrm{OR}=1.79$, $95 \% \mathrm{CI}=1.40-2.30), \mathrm{AD} / \mathrm{MCI}(p<0.001 ; \mathrm{OR}=1.76,95 \% \mathrm{CI}=1.40-2.22)$ and control groups $(p<0.001 ; \mathrm{OR}=1.97$, 


\begin{tabular}{|c|c|c|c|c|c|c|}
\hline & Seoul $(n=1099)$ & Kaohsiung $(n=363)$ & Singapore $(n=214)$ & Beijing $(n=190)$ & Manilla $(n=180)$ & $p^{*}$ \\
\hline Age, year, mean \pm SD (min; max) & $77.45 \pm 7.56(37 ; 99)$ & $80.23 \pm 8.01(58 ; 99)$ & $70.92 \pm 9.38(49 ; 91)$ & $72.46 \pm 7.51(46 ; 88)$ & $73.82 \pm 9.58(50 ; 92)$ & $p<0.001^{\mathrm{a}, \mathrm{d}, \mathrm{e}, \mathrm{f}, \mathrm{g}, \mathrm{h}, \mathrm{i}, \mathrm{j}}$ \\
\hline Education, year, mean \pm SD (min; max $)$ & $7.33 \pm 5.33(0 ; 20)$ & $6.40 \pm 5.21(0 ; 20)$ & $7.83 \pm 4.83(0 ; 22)$ & $12.25 \pm 4.68(0 ; 21)$ & $13.98 \pm 3.40(0 ; 20)$ & $p<0.001^{\mathrm{a}, \mathrm{b}, \mathrm{b}, \mathrm{d}, \mathrm{e}, \mathrm{g}, \mathrm{h}, \mathrm{i}, \mathrm{j}}$ \\
\hline Female; $n(\%)$ & $723(65.8 \%)$ & $247(68.0 \%)$ & $117(54.7 \%)$ & $123(64.7 \%)$ & $99(55.0 \%)$ & $p=0.001^{\mathrm{e}, \mathrm{f}, \mathrm{i}, \mathrm{j}}$ \\
\hline Hypertension; $n(\%)$ & $491(44.7 \%)$ & $167(47.2 \%)$ & $120(59.7 \%)$ & $63(36.2 \%)$ & $113(63.1 \%)$ & $p<0.001^{\mathrm{a}, \mathrm{b}, \mathrm{c}, \mathrm{i}, \mathrm{j}}$ \\
\hline Diabetes mellitus; $n(\%)$ & $282(25.7 \%)$ & $82(23.7 \%)$ & $64(31.8 \%)$ & $27(15.5 \%)$ & $65(36.1 \%)$ & $p<0.001^{\mathrm{a}, \mathrm{b}, \mathrm{c}, \mathrm{e}, \mathrm{i}}$ \\
\hline Hyperlipidemia; $n(\%)$ & $215(19.6 \%)$ & 1 & $119(59.2 \%)$ & $57(33.1 \%)$ & $70(38.9 \%)$ & $p<0.001^{\text {a,ce, e, }, \mathrm{h}}$ \\
\hline Ischemic heart disease; $n(\%)$ & $122(11.1 \%)$ & $3(4.1 \%)$ & $20(9.4 \%)$ & $25(14.4 \%)$ & $35(19.6 \%)$ & $p=0.002^{\mathrm{e}, \mathrm{h}, \mathrm{i}}$ \\
\hline Atrial fibrillation; $n(\%)$ & $23(2.6 \%)$ & $3(4.1 \%)$ & $6(2.8 \%)$ & $6(3.4 \%)$ & $4(2.2 \%)$ & $p=0.900$ \\
\hline Smoker (either current or past); $n$ (\%) & $41(19.0 \%)$ & $19(18.1 \%)$ & $14(6.5 \%)$ & $31(17.9 \%)$ & $54(30.0 \%)$ & $p<0.001^{\mathrm{c}, \mathrm{fh}, \mathrm{j}}$ \\
\hline MMSE, mean \pm SD (min; max) & $18.97 \pm 5.58(0 ; 30)$ & $15.38 \pm 6.38(0 ; 28)$ & $20.40 \pm 5.58(3 ; 30)$ & $20.42 \pm 6.46(1 ; 30)$ & $21.78 \pm 6.08(5 ; 30)$ & $p<0.001^{\text {a,de,f, }, \mathrm{g}, \mathrm{i}, \mathrm{j}}$ \\
\hline $\mathrm{WML}=0$ & $182(16.6 \%)$ & $57(15.7 \%)$ & $15(7.0 \%)$ & $2(1.1 \%)$ & $12(6.7 \%)$ & $p<0.001^{\mathrm{a}, \mathrm{b}, \mathrm{b}, \mathrm{d}, \mathrm{e}, \mathrm{f}, \mathrm{g}, \mathrm{h}, \mathrm{i}, \mathrm{j}}$ \\
\hline $\mathrm{WML}=1$ & $570(51.9 \%)$ & $94(25.9 \%)$ & $75(35.0 \%)$ & $118(62.1 \%)$ & $134(74.4 \%)$ & \\
\hline $\mathrm{WML}=2$ & $228(20.7 \%)$ & $90(24.8 \%)$ & $88(41.1 \%)$ & $61(32.1 \%)$ & $26(14.4 \%)$ & \\
\hline $\mathrm{WML}=3$ & $119(10.8 \%)$ & $122(33.6 \%)$ & $36(16.8 \%)$ & $9(4.7 \%)$ & $8(4.4 \%)$ & \\
\hline $\begin{array}{l}\text { Prevalence of moderate to severe WML } \\
(\geq 2) ; n(\%)\end{array}$ & $347(31.6 \%)$ & $212(58.4 \%)$ & $124(57.9 \%)$ & $70(36.8 \%)$ & $34(18.9 \%)$ & $p<0.001^{\mathrm{b}, \mathrm{c}, \mathrm{d}, \mathrm{e}, \mathrm{f}, \mathrm{g}, \mathrm{h}, \mathrm{i}}$ \\
\hline
\end{tabular}

Table 3. Clinical demographics of the AD/MCI group. This table showed the clinical demographics of the $\mathrm{AD} / \mathrm{MCI}$ group. ${ }^{\mathrm{a} B e i j i n g}$ versus Seoul. ${ }^{\mathrm{b}}$ Beijing versus Manilla. ${ }^{\mathrm{c} B e i j i n g}$ versus Singapore. ${ }^{\mathrm{d}}$ Beijing versus

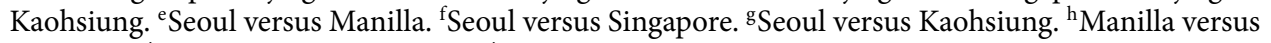
Singapore. ${ }^{i}$ Manilla versus Kaohsiung. ${ }^{j}$ Singapore versus Kaohsiung. MMSE Mini-mental state examination. $W M L$ White matter lesion. ${ }^{\star}$ Bonferroni correction is applied to the alpha $(\alpha=0.005)$.

\begin{tabular}{|c|c|c|c|c|}
\hline & Hong Kong $(n=850)$ & Singapore $(n=832)$ & Seoul $(n=115)$ & $p^{*}$ \\
\hline Age, year, mean \pm SD (min; $\max )$ & $71.37 \pm 5.09(64 ; 89)$ & $69.96 \pm 6.46(60 ; 88)$ & $64.72 \pm 5.31(60 ; 79)$ & $p<0.001^{\mathrm{a}, \mathrm{b}, \mathrm{c}}$ \\
\hline Education, year, mean \pm SD $(\min ; \max )$ & $7.89 \pm 4.91(0 ; 22)$ & $6.07 \pm 4.52(0 ; 22)$ & $10.12 \pm 3.37(6 ; 18)$ & $p<0.001^{\mathrm{a}, \mathrm{b}, \mathrm{c}}$ \\
\hline Female; $n(\%)$ & $524(61.6 \%)$ & $434(52.2 \%)$ & $66(57.4 \%)$ & $p<0.001^{\mathrm{b}}$ \\
\hline Hypertension; $n(\%)$ & $519(61.1 \%)$ & $669(80.4 \%)$ & $32(27.8 \%)$ & $p<0.001^{\mathrm{a}, \mathrm{b}, \mathrm{c}}$ \\
\hline Diabetes mellitus; $n(\%)$ & $202(23.8 \%)$ & $311(37.4 \%)$ & $16(13.9 \%)$ & $p<0.001^{\mathrm{b}, \mathrm{c}}$ \\
\hline Hyperlipidemia; $n(\%)$ & $264(31.1 \%)$ & $627(75.4 \%)$ & $18(15.7 \%)$ & $p<0.001^{\mathrm{a}, \mathrm{b}, \mathrm{c}}$ \\
\hline Smoker (either current or past); $n$ (\%) & $160(18.8 \%)$ & $224(26.9 \%)$ & $35(30.4 \%)$ & $p<0.001^{\mathrm{a}, \mathrm{b}}$ \\
\hline MMSE, mean \pm SD $(\min ; \max )$ & $26.94 \pm 2.36(19 ; 30)$ & $23.87 \pm 3.80(2 ; 30)$ & $28.21 \pm 1.38(23 ; 30)$ & $p<0.001^{\mathrm{a}, \mathrm{b}, \mathrm{c}}$ \\
\hline $\mathrm{WML}=0$ & $170(20.0 \%)$ & $25(3.0 \%)$ & $63(54.8 \%)$ & $p<0.001^{\mathrm{a}, \mathrm{b}, \mathrm{c}}$ \\
\hline $\mathrm{WML}=1$ & $435(51.2 \%)$ & $412(49.5 \%)$ & $33(28.7 \%)$ & \\
\hline $\mathrm{WML}=2$ & $187(22.0 \%)$ & $332(39.9 \%)$ & $14(12.2 \%)$ & \\
\hline $\mathrm{WML}=3$ & $58(6.8 \%)$ & $63(7.6 \%)$ & $5(4.3 \%)$ & \\
\hline Prevalence of moderate to severe WML $(\geq 2) ; n(\%)$ & $245(28.8 \%)$ & $395(47.5 \%)$ & $19(16.5 \%)$ & $p<0.001^{\mathrm{a}, \mathrm{b}, \mathrm{c}}$ \\
\hline
\end{tabular}

Table 4. Clinical demographics of the control group. This table showed the clinical demographics of the control group. MMSE Mini-mental state examination, WML White matter lesion. ${ }^{a}$ Hong Kong versus Seoul. ${ }^{b}$ Hong Kong versus Singapore. ${ }^{c}$ Seoul versus Singapore. ${ }^{*}$ Bonferroni correction is applied to the alpha $(\alpha=0.0167)$.

95\% CI $=1.52-2.55$ ) (Table 5). Strength of association between hypertension and moderate-to-severe WML decreased with increasing age. Similar pattern was observed in all subjects, the stroke/TIA, AD/MCI and control groups (see Supplementary Tables S4-S7).

The association between hyperlipidemia and moderate-to-severe WML was significant in all subjects $(p=0.012 ; \mathrm{OR}=1.19,95 \% \mathrm{CI}=1.04-1.36)$, as well as in those in the $61-70$ age range $(p=0.049 ; \mathrm{OR}=1.28,95 \%$ $\mathrm{CI}=1.00-1.64$ ), (Table 5 and Supplementary Table S4). However, the association in the 61-70 age group did not survive Bonferroni correction for multiple comparison. There was no significant association between diabetes mellitus and moderate-to-severe WML in all disease groups.

The risk of moderate-to-severe WML in different Asian regions. To examine the variations of WML in different regions and account for sampling bias in each region, the regression was performed with adjustment for age, sex and level of education, Asian regions, disease groups, and imaging modality (see Table 5). Using Hong Kong as a reference center, Bandung showed a higher risk of having moderate-to-severe WML $(p<0.001$; $\mathrm{OR}=45.41,95 \% \mathrm{CI}=25.72-80.19)$ while Manila showed a lower risk $(p<0.001 ; \mathrm{OR}=0.26,95 \% \mathrm{CI}=0.15-0.44)$ when all groups were combined. 


\begin{tabular}{|c|c|c|c|c|}
\hline \multirow[b]{2}{*}{ Dependent variables } & \multirow[b]{2}{*}{ OR } & \multicolumn{2}{|l|}{$95 \% \mathrm{CI}$} & \multirow[b]{2}{*}{$p^{*}$} \\
\hline & & Lower & Upper & \\
\hline \multicolumn{5}{|l|}{ All subjects $(n=5215)$} \\
\hline Age & 1.07 & 1.06 & 1.08 & $<0.001$ \\
\hline Female & 1.04 & 0.91 & 1.18 & 0.608 \\
\hline Level of education & 0.99 & 0.98 & 1.00 & 0.087 \\
\hline HT & 1.81 & 1.58 & 2.08 & $<0.001$ \\
\hline $\mathrm{DM}$ & 1.10 & 0.96 & 1.26 & 0.163 \\
\hline HLD & 1.19 & 1.04 & 1.36 & 0.012 \\
\hline Hong Kong (reference) & 1 & 1 & 1 & $<0.001$ \\
\hline Beijing & 0.95 & 0.60 & 1.51 & 0.816 \\
\hline Bangkok & 2.08 & 1.38 & 3.14 & 0.001 \\
\hline Bandung & 45.41 & 25.72 & 80.19 & $<0.001$ \\
\hline Seoul Korea & 0.48 & 0.34 & 0.68 & $<0.001$ \\
\hline Manilla & 0.26 & 0.15 & 0.44 & $<0.001$ \\
\hline Singapore & 1.81 & 1.49 & 2.20 & $<0.001$ \\
\hline Kaohsiung & 0.48 & 0.35 & 0.66 & $<0.001$ \\
\hline Control (reference) & I & 1 & 1 & $<0.001$ \\
\hline $\mathrm{AD} / \mathrm{MCI}$ & 1.54 & 1.14 & 2.08 & 0.005 \\
\hline Stroke/TIA & 1.90 & 1.49 & 2.43 & $<0.001$ \\
\hline CT (reference) & I & 1 & 1 & $<0.001$ \\
\hline MRI $1.5 \mathrm{~T}$ & 1.37 & 1.00 & 1.86 & 0.047 \\
\hline MRI 3 T & 1.75 & 1.33 & 2.32 & $<0.001$ \\
\hline \multicolumn{5}{|l|}{ Stroke/TIA (n= 1786) } \\
\hline Age & 1.07 & 1.06 & 1.08 & $<0.001$ \\
\hline Female & 1.26 & 1.01 & 1.57 & 0.040 \\
\hline Level of education & 0.99 & 0.97 & 1.02 & 0.493 \\
\hline HT & 1.79 & 1.40 & 2.30 & $<0.001$ \\
\hline $\mathrm{DM}$ & 1.08 & 0.87 & 1.35 & 0.482 \\
\hline HLD & 1.21 & 0.97 & 1.51 & 0.092 \\
\hline Hong Kong (reference) & 1 & 1 & 1 & 0.000 \\
\hline Bangkok & 1.76 & 1.15 & 2.70 & 0.010 \\
\hline Bandung & 44.01 & 24.28 & 79.77 & $<0.001$ \\
\hline Singapore & 0.72 & 0.44 & 1.19 & 0.200 \\
\hline Kaohsiung & 0.37 & 0.26 & 0.52 & $<0.001$ \\
\hline CT (reference) & 1 & 1 & 1 & $<0.001$ \\
\hline MRI $1.5 \mathrm{~T}$ & 1.23 & 0.89 & 1.70 & 0.216 \\
\hline MRI $3 \mathrm{~T}$ & 2.19 & 1.59 & 3.00 & $<0.001$ \\
\hline \multicolumn{5}{|l|}{$\mathrm{AD} / \mathrm{MCI}(\mathrm{n}=1633)$} \\
\hline Age & 1.06 & 1.05 & 1.08 & $<0.001$ \\
\hline Female & 0.81 & 0.63 & 1.04 & 0.095 \\
\hline Level of education & 1.01 & 0.98 & 1.03 & 0.643 \\
\hline HT & 1.76 & 1.40 & 2.22 & $<0.001$ \\
\hline $\mathrm{DM}$ & 1.07 & 0.83 & 1.38 & 0.602 \\
\hline HLD & 1.12 & 0.86 & 1.45 & 0.404 \\
\hline Beijing (reference) & 1 & 1 & 1 & $<0.001$ \\
\hline Seoul Korea & 0.55 & 0.38 & 0.81 & 0.002 \\
\hline Manilla & 0.29 & 0.17 & 0.48 & $<0.001$ \\
\hline Singapore & 2.71 & 1.69 & 4.35 & $<0.001$ \\
\hline CT (reference) & 1 & 1 & 1 & 0.993 \\
\hline MRI $1.5 \mathrm{~T}$ & 1 & 1 & 1 & 0.999 \\
\hline MRI 3 T & 1 & 1 & 1 & 0.999 \\
\hline \multicolumn{5}{|l|}{ Controls $(n=1796)$} \\
\hline Age & 1.10 & 1.08 & 1.12 & $<0.001$ \\
\hline Female & 0.94 & 0.75 & 1.18 & 0.591 \\
\hline Level of education & 0.98 & 0.96 & 1.01 & 0.184 \\
\hline HT & 1.97 & 1.52 & 2.55 & $<0.001$ \\
\hline
\end{tabular}




\begin{tabular}{|l|l|l|l|r|}
\hline & & \multicolumn{2}{|l|}{$95 \%$ CI } & \multirow{2}{*}{} \\
\cline { 3 - 5 } Dependent variables & OR & Lower & Upper & \multicolumn{1}{|c|}{$\boldsymbol{p}^{*}$} \\
\hline DM & 1.15 & 0.91 & 1.45 & 0.233 \\
\hline HLD & 1.12 & 0.88 & 1.43 & 0.356 \\
\hline Hong Kong (reference) & $/$ & $/$ & $/$ & $<0.001$ \\
\hline Seoul Korea & 1.13 & 0.65 & 1.95 & 0.671 \\
\hline Singapore & 2.18 & 1.71 & 2.79 & $<0.001$ \\
\hline
\end{tabular}

Table 5. Risk factors of moderate-to-severe white matter lesion. This table shows the vascular risk factors contributing to moderate-to-severe white matter lesion. Age, sex, level of education, Asian cities, disease groups, and image modality are entered as covariates in each regression model as appropriate. DM Diabetes mellitus, HLD Hyperlipidemia, HT Hypertension. ${ }^{\star}$ Bonferroni correction is applied to the alpha $(\alpha=0.0125)$.

\begin{tabular}{|c|c|c|c|c|}
\hline \multirow[b]{2}{*}{$\mathbf{n}$} & \multirow[b]{2}{*}{$\beta$} & \multicolumn{2}{|c|}{$95 \%$ CI for B } & \multirow[b]{2}{*}{$p^{*}$} \\
\hline & & Lower & Upper & \\
\hline \multicolumn{5}{|c|}{ All subjects } \\
\hline 5519 & -0.052 & -0.945 & -0.415 & $<0.001$ \\
\hline \multicolumn{5}{|c|}{ Stroke/TIA } \\
\hline 1765 & -0.056 & -1.316 & -0.258 & 0.004 \\
\hline \multicolumn{5}{|c|}{$\mathrm{AD} / \mathrm{MCI}$} \\
\hline 1958 & -0.079 & -1.478 & -0.517 & $<0.001$ \\
\hline \multicolumn{5}{|c|}{ Controls } \\
\hline 1796 & -0.064 & -0.735 & -0.191 & 0.001 \\
\hline
\end{tabular}

Table 6. Association between MMSE and moderate-to-severe white matter lesion. This table shows the association between moderate-to-severe white matter lesion contributing to cognition. Age, sex, level of education, Asian cities and hypertension are entered as covariates in each regression model. MMSE Minimental state examination. ${ }^{*}$ Bonferroni correction is applied to the alpha $(\alpha=0.0125)$.

When focusing on the stroke/TIA group, Bandung showed a higher risk of having moderate-to-severe WML $(p<0.001 ; \mathrm{OR}=44.01,95 \% C I=24.28-79.77)$ while Kaohsiung showed a lower risk $(p=0.001 ; \mathrm{OR}=0.37,95 \%$ $C I=0.26-0.52)$ compared to Hong Kong. Using Beijing as a reference center, Singapore showed a higher risk of having moderate-to-severe WML $(p<0.001 ; \mathrm{OR}=2.71,95 \% C I=1.69-4.35)$ while Manila showed a lower risk of WML $(p<0.001 ; \mathrm{OR}=0.29,95 \% C I=0.17-0.48)$ in the AD/MCI group. Finally, Singapore showed a higher risk of having moderate-to-severe WML compared to Hong Kong and Seoul $(p<0.001 ; \mathrm{OR}=2.18,95 \% \mathrm{CI}=1.71-2.79)$.

Moderate-to-severe WML and cognition. Multiplelinear regression analyses showed that the presence of moderate-to-severe WML was significantly associated with lower MMSE scores among all subjects and among each disease group (see Table 6). Overall, the MMSE was 20.6 \pm 6.2 in those with moderate-to-severe WML $(n=2529)$ compared to $22.5 \pm 6.5$ in those none-or-mild WML $(n=3621)$. Further, subgroup analysis showed that MMSE was approximately 2 points lower in those with moderate-to-severe WML compared to those without. The MMSE scores in the moderate-to-severe WML and none-or-mild WML were $20.4 \pm 6.9$ and $22.6 \pm 7.0$ respectively in the stroke/TIA group; $17.5 \pm 6.2$ and $19.7 \pm 5.9$ respectively in the AD/MCI group; and $24.5 \pm 3.9$ and $26.3 \pm 3.0$ respectively in the control group. Subjects with stroke/TIA and dementia had a higher prevalence of moderate-to-severe WML than those with stroke/TIA but without dementia (51.7\% vs $40.7 \%$ ). Subjects with $\mathrm{AD}$ dementia also had a higher prevalence of moderate-to-severe WML than those with MCI (41.2\% vs $25.3 \%)$.

\section{Discussion}

This is the first multi-center study to formally assess the prevalence of moderate-to-severe WML in stroke/TIA and $\mathrm{AD} / \mathrm{MCI}$, in different age groups, and from 9 Asian cohorts. The prevalence of moderate-to-severe WML was higher in stroke/TIA (43.3\%) than that in $\mathrm{AD} / \mathrm{MCI}(38.2 \%), \mathrm{X}_{(1)}^{2}=10.6, p=0.001$. Moderate-to-severe WML was associated with hypertension, hyperlipidemia, and a lower MMSE score.

In this study, we primarily reported the prevalence of moderate-to-severe WML, rather than any presence of WML. Previous studies that included any presence of WML (i.e. including those with mild WML as well) reported a much higher prevalence of WML, reaching $81.4 \%$ in the community ${ }^{37}$. We recorded only moderateto-severe WML because previous studies showed that only those with moderate-to-severe WML as defined by a grade 2 or above in the global rating of Fazekas or ARMCW scale were associated with increased risk of incident cognitive decline. Although mild or focal/punctate WML may still represent early or minor SVD, previous studies showed that it had no or minimal clinical relevance, while longitudinal studies revealed no progression of mild WML over time ${ }^{35}$. Whereas, early confluent to confluent WML will likely progress in size and is clinically 
relevant ${ }^{15,18,20,35}$. In cohorts where the prevalence of mild WML is high and that of moderate-to-severe WML is low, reporting any presence of WML may overestimate the severity of CSVD.

WML in stroke/TIA. Despite the mean age of the stroke/TIA group was the youngest $(67.7 \pm 12.2$ years old $)$ when compared with the $\mathrm{AD} / \mathrm{MCI}$ group $(76.3 \pm 8.7$ years old) and controls $(70.3 \pm 6.0$ years old), the stroke/ TIA group had a higher prevalence of moderate-to-severe WML to that of AD/MCI group as a whole and at each age group, with a prevalence of $32.2 \%$ for age $\leq 60$ that increased to $64.2 \%$ for subjects older than 80 years (Fig. 2). The high prevalence of moderate-to-severe WML in stroke/TIA was most probably explained to a large extent by the fact that both stroke/TIA and WML shared a strong association with cardiovascular risk factors, in particular hypertension ${ }^{38}$. The most alarming finding was observed in Bandung, Indonesia where the prevalence of moderate-to-severe WML is highest among all cities. In particular, hypertension (81.5\%) in this group was high despite the relatively young mean age ( 59 years old). In addition, the Indonesian cohort provided only CT imaging and hence we might have even underestimated the prevalence of moderate-to-severe WML, as CT is less sensitive in the detection of WML relative to MRI. This is indeed the first study investigating the prevalence of WML in Indonesia, which showed a very high prevalence of WML in this region, alongside a high prevalence of hypertension.

WML in AD/MCl. The prevalence of moderate-to-severe WML in the AD/MCI group was lower than that in stroke/TIA across all age distributions by about $12-15 \%$. Still, the prevalence ranged from $20 \%$ for age $\leq 60$ to almost $50 \%$ for subjects older than 80 years old. The prevalence of HT and other cardiovascular risk factors were lower in $\mathrm{AD} / \mathrm{MCI}$ than that in the stroke/TIA group, which might at least partially explain the lower prevalence of WML in $\mathrm{AD} / \mathrm{MCI}$ than in the stroke/TIA group. Another interesting observation was seen in Manila, despite having a large proportion of patients having hypertension (63.1\%) and being a current or a past smoker (30\%), only $18.9 \%$ of the patients in Manila had the lowest prevalence of moderate-to-severe WML. Note that all subjects in the Manila cohort had MRI, and hence, under-reporting of WML was not likely. Whether Filipinos are less susceptible to the development of WML in association with hypertension requires further investigation ${ }^{39}$.

WML in the control group. Our previous report on the prevalence of CSVD in Asian communities did not report specifically the prevalence of moderate-to-severe WML according to different age groups ${ }^{27}$. In the current study, the prevalence of moderate-to-severe WML rose from $28.1 \%$ at $61-70$ years old to $65.2 \%$ at $\geq 81$ years old. Of note, this prevalence of WML is even slightly higher than the prevalence rates of amyloid positivity reported previously among subjects with normal cognition, which vary from around $20 \%$ at 70 years old to slightly above $40 \%$ at 90 years old ${ }^{40}$. Among the three cities with community subjects, Singapore recorded the highest prevalence of moderate-to-severe WML despite a mean age of only 69.96 years old, which could again be related to the fact that the Singapore group had the highest prevalence of hypertension (80.4\%) and hyperlipidemia (75.4\%) relative to other cities.

Previous studies on the prevalence of WML have been conducted mainly among Europe and North America. Existing studies showed that WML is almost endemic among the elderly. In the general population, the prevalence of WML was 39\% in the Helsinki Aging Brain Study. In particular, WML prevalence was detected in 21\% in those $<75$ years old and $65 \%$ in those $\geq 75$ years old ${ }^{41}$. In the oldest-old (80-90 years), either subcortical and periventricular WML was detected in $\geq 95 \%$ of the subjects in the Rotterdam Scan Study ${ }^{42}$. When assessing WML longitudinally within the same cohort, the Austrian Stroke Prevention Study showed that the median increase of WML volume to reach early confluent to confluent WML was $36 \%$ at the 3 -year follow-up and $58 \%$ at the 6 -year follow-up ${ }^{43}$. The incidence and prevalence of WML vary depending on numerous factors including the age, sex of the subject, whether the subject is healthy or has experienced stroke or dementia, the quality of image acquired, as well as the WML rating method. Thus, direct comparisons or interpretation of the results remain challenging.

Association between WML, vascular risk factors and global cognition. We found that the strength of association between hypertension and moderate-to-severe WML decreased with increasing age, which suggested that the effects of blood pressure-lowering therapy may be more effective in the younger age group. While among older patients, factors other than high blood pressure (e.g. impaired autoregulation) may have a greater contribution to $\mathrm{WML}^{44}$. Note further that the strength of association between hypertension and WML in the $\mathrm{AD} / \mathrm{MCI}$ group $(p<0.001 ; \mathrm{OR}=1.76,95 \% \mathrm{CI}=1.40-2.22)$ was similar to that of in the stroke/TIA $(p<0.001$; $\mathrm{OR}=1.79,95 \% \mathrm{CI}=1.40-2.30)$ or control groups $(p<0.001 ; \mathrm{OR}=1.97,95 \% \mathrm{CI}=1.52-2.55)$, suggesting that moderate-to-severe WML observed in subjects with $\mathrm{AD} / \mathrm{MCI}$ may have a significant vascular component.

Previous studies showed conflicting results concerning the association between hyperlipidemia and $\mathrm{WML}^{45,46}$. The present study with a large sample size of approximately 6000 subjects did show a small yet significant association between hyperlipidemia and moderate-to-severe WML. The strength of association was less than that for hypertension. Similar to most previous studies, we could not demonstrate an association between DM and WML. Note, however, that other studies showed that DM was related to measures of lacunes, rather than to WML ${ }^{47-49}$.

Above all, we have shown that moderate-to-severe WML was associated with worse cognitive performance in stroke/TIA, AD/MCI and controls. MMSE of those with moderate-to-severe WML was approximately 2 points lower compared to those without moderate-to-severe WML among all subjects and among each of the clinical groups.

Strengths and limitations. The strength of this study includes the large sample size, the inclusion of the $\mathrm{AD} / \mathrm{MCI}$ and stroke/TIA groups that are known with a high prevalence of moderate-to-severe WML, as well as a large control group for comparison. Further, this is the first joint international effort with standardized meas- 
urement of the WML burden across multiple clinical cohorts in different Asian cities. In addition, analyses in this study were also adjusted with confounders such as age, sex, education, vascular risk factors, disease groups, and different Asian cities.

However, there are certain limitations in this study. First, we acknowledge that certain biases were introduced in the study. Similar to any multi-center study, the pooled sample may contain a more heterogeneous dataset across centers, as well as a potential source of sampling bias from non-random recruitment. However, we have set the study-specific inclusion and exclusion criteria and used the same neuropsychological screening test (MMSE). Survival bias may be introduced as the patients enrolled in the study could have been biased to the younger group who had better cognitive function, and milder chronic brain changes. This could lead to an underestimation of the true magnitude of the prevalence of WML in each city, as well as the association between WML, vascular risk factors, and cognition.

Second, we have only used a brief screening test to measure the overall cognitive function. MMSE is not sensitive in detecting executive dysfunction or slow processing speed that are predominantly affected by CSVD. Further, the MMSE was administered in various languages and cultures specific to the local population and there may be characteristics differences in each version ${ }^{50}$. Third, we acknowledge that Fazekas and ARWMC are two different visual rating scales for WML. Although the scales are different, a cut-off at $\geq 2$ in both scales was selected as they both indicated a certain severity of WML that is progressive, and thus, malignant ${ }^{43}$, and lead to devastating outcomes such as global functional decline or delayed-onset dementia ${ }^{18,20,51}$. The Hong Kong community dataset with both Fazekas and ARWMC global ratings showed that the correlation between the scales was high $(p<0.0001 ; r=0.943)$.

Forth, WML can be associated with inflammation, cerebral amyloid angiopathy, or neurodegenerative disease secondary to Wallerian degeneration, which we were unable to differentiate in this study. Fifth, the use of antihypertensive medications may delay the progression of $\mathrm{WML}^{52}$. Most subjects with hypertension included in this study received treatment. However, there is no sufficient data collected to provide further information on how well the hypertension was controlled or types of medication used. Similarly, the management of hypertension may vary among regions and may confer different risks. Future studies should take more detailed information about types of medication used and whether hypertension was controlled, in addition to objective measures of systolic and diastolic blood pressure. Sixth, although we had included data from Bengaluru India to investigate the prevalence of WML and vascular risk factors, the small sample size prevented us from entering the data in regression analyses. Finally, other CSVD imaging markers such as lacunes, cerebral microbleeds, enlarged perivascular space, and microinfarcts were not assessed and may have impact on the overall clinical manifestation.

Implications. Our findings that there were high burden of CSVD/WMLs in stroke/TIA, AD/MCI, community controls, and specific cities (e.g. Indonesian, Singaporean), and that it was associated with hypertension, hyperlipidemia, and poor global cognition. These findings have huge implications in the management, research and public health strategies for preventing dementia in Asia. Strategies that can improve management of high blood pressure and lipids at both population and individual levels cannot only prevent stroke but also potentially prevent or delay the development and progression of subclinical CSVD/WML. Recent clinical trials of large sample sizes and long durations did show positive effects of aggressive blood pressure lowering and use of statins upon reducing the progression of WML, along with possible benefits upon cognition ${ }^{53-57}$. Strategies targeting CSVD should also incorporate other measures relevant to dementia prevention (e.g. reduce air pollution, limit alcohol, avoid smoking, provide primary and secondary education, introduce a healthy diet, reduce obesity and diabetes, increase physical exercise, and improve sleep quality, etc.) to achieve the maximal effect in reducing dementia burden. Management of hypertension, in particular, should start in mid-life as the evolution of WML from no to severe WML may take more than a decade. Such comprehensive strategies if can be implemented aggressively and efficiently in cities with a high burden of vascular risk factors and CSVD (e.g. Indonesia), the effect size in preventing dementia cases in these cities may be more pronounced. In addition to primary preventive strategies, we propose that more clinical trials should target individuals who already harbor moderateto-severe CSVD/WML at different clinical contexts, e.g. poststroke/TIA with cognitive impairment (without dementia), MCI (prodromal AD), or even in "preclinical CSVD" dementia- and stroke-free individuals, as they are at high risk of further cognitive decline or dementia. Preferably, such trials should include Asians so that findings from these trials can be generalized to Asians as well.

Received: 8 December 2020; Accepted: 13 May 2021

Published online: 02 June 2021

\section{References}

1. Prince, M. J., Wimo, A., Guerchet, M. M., Ali, G. C., Wu, Y.-T., \& Prina, M.. World Alzheimer Report 2015 - The Global Impact of Dementia: An analysis of prevalence, incidence, cost and trends. London: Alzheimer's Disease International, 84 (2015).

2. Toledo, J. B. et al. Contribution of cerebrovascular disease in autopsy confirmed neurodegenerative disease cases in the National Alzheimer's Coordinating Centre. Brain 136, 2697-2706 (2013).

3. Wardlaw, J. M. et al. Neuroimaging standards for research into small vessel disease and its contribution to ageing and neurodegeneration. Lancet Neurol. 12, 822-838. https://doi.org/10.1016/S1474-4422(13)70124-8 (2013).

4. Smith, E. E., Schneider, J. A., Wardlaw, J. M. \& Greenberg, S. M. Cerebral microinfarcts: the invisible lesions. Lancet Neurol. 11, 272-282 (2012).

5. Jokinen, H. et al. Post-stroke cognitive impairment is common even after successful clinical recovery. Eur. J. Neurol. 22, 1288-1294. https://doi.org/10.1111/ene.12743 (2015). 
6. Wen, H. M. et al. Effect of white matter changes on cognitive impairment in patients with lacunar infarcts. Stroke https://doi.org/ 10.1161/01.str.0000133686.29320.58 (2004).

7. Wang, Z. et al. Cerebral microbleeds and cognitive function in ischemic stroke or transient ischemic attack patients. Dement. Geriatr. Cogn. Disord. 40, 130-136 (2015).

8. Wang, Z. et al. Risk factors and cognitive relevance of cortical cerebral microinfarcts in patients with ischemic stroke or TIA. Stroke 47, 2450-2455 (2016).

9. Mok, V. C. T. et al. Early-onset and delayed-onset poststroke dementia - revisiting the mechanisms. Nat. Rev. Neurol. 13, 148-159. https://doi.org/10.1038/nrneurol.2017.16 (2017).

10. De Groot, J. C. et al. Cerebral white matter lesions and cognitive function: the Rotterdam Scan Study. Ann. Neurol. Off. J. Am. Neurol. Assoc. Child Neurol. Soc. 47, 145-151 (2000).

11. Kao, Y.-H., Chou, M.-C., Chen, C.-H. \& Yang, Y.-H. White matter changes in patients with Alzheimer's disease and associated factors. J. Clin. Med. 8, 167 (2019).

12. Mok, V. C. T. et al. Cognitive impairment and functional outcome after stroke associated with small vessel disease. J. Neurol. Neurosurg. Psychiatry 75, 560-566. https://doi.org/10.1136/jnnp.2003.015107 (2004).

13. Pendlebury, S. T. \& Rothwell, P. M. Prevalence, incidence, and factors associated with pre-stroke and post-stroke dementia: a systematic review and meta-analysis. Lancet Neurol. 8, 1006-1018 (2009).

14. Prasad, K., Wiryasaputra, L., Ng, A. \& Kandiah, N. White matter disease independently predicts progression from mild cognitive impairment to Alzheimer's disease in a clinic cohort. Dement. Geriatr. Cogn. Disord. 31, 431-434. https://doi.org/10.1159/00033 0019 (2011)

15. Kim, S. et al. Periventricular white matter hyperintensities and the risk of dementia: a CREDOS study. Int. Psychogeriatr. 27, 2069 (2015).

16. Alosco, M. L. et al. A clinicopathological investigation of white matter hyperintensities and Alzheimer's disease neuropathology. J. Alzheimer's Dis. JAD 63, 1347-1360. https://doi.org/10.3233/JAD-180017 (2018).

17. Lee, S. et al. White matter hyperintensities are a core feature of Alzheimer's disease: evidence from the dominantly inherited Alzheimer network. Ann. Neurol. 79, 929-939. https://doi.org/10.1002/ana.24647 (2016).

18. Ding, D. et al. White matter hyperintensity predicts the risk of incident cognitive decline in community dwelling elderly. J. Alzheimers Dis. 61, 1333-1341 (2018).

19. Debette, S. \& Markus, H. S. The clinical importance of white matter hyperintensities on brain magnetic resonance imaging: systematic review and meta-analysis. BMJ 341, 1-9. https://doi.org/10.1136/bmj.c3666 (2010).

20. Inzitari, D. et al. Changes in white matter as determinant of global functional decline in older independent outpatients: three year follow-up of LADIS (leukoaraiosis and disability) study cohort. BMJ https://doi.org/10.1136/bmj.b2477 (2009).

21. Jorgensen, D. R. et al. A population neuroscience approach to the study of cerebral small vessel disease in midlife and late life: an invited review. Am. J. Physiol. Heart Circ. Physiol. 314, H1117-H1136 (2018).

22. Marini, S., Anderson, C. D. \& Rosand, J. Genetics of cerebral small vessel disease. Stroke 51, 12-20 (2020)

23. Mok, V. et al. Race-ethnicity and cerebral small vessel disease-comparison between Chinese and White populations. Int. J. Stroke 9, 36-42 (2014).

24. Hiremath, N., Kate, M., Mohimen, A., Kesavadas, C. \& Sylaja, P. N. Risk factors of white matter hyperintensities in South Asian patients with transient ischemic attack and minor stroke. Neuroradiology https://doi.org/10.1007/s00234-020-02429-5 (2020).

25. Ren, X.-M. et al. White matter lesions predict recurrent vascular events in patients with transient ischemic attacks. Chin. Med. J. (Engl.) 131, 130-136. https://doi.org/10.4103/0366-6999.222341 (2018).

26. Lee, S.-J. et al. The leukoaraiosis is more prevalent in the large artery atherosclerosis stroke subtype among Korean patients with ischemic stroke. BMC Neurol. 8, 31-31. https://doi.org/10.1186/1471-2377-8-31 (2008).

27. Hilal, S. et al. Prevalence, risk factors and consequences of cerebral small vessel diseases: data from three Asian countries. J. Neurol. Neurosurg. Psychiatry 88, 669 (2017).

28. McKhann, G. et al. Clinical diagnosis of Alzheimer's disease. Report of the NINCDS-ADRDA Work Group* under the auspices of Department of Health and Human Services Task Force on Alzheimer's Disease 34, 939-939, https://doi.org/10.1212/wnl.34.7.939 (1984).

29. Sacco, R. L. et al. An updated definition of stroke for the 21st century: a statement for healthcare professionals from the American Heart Association/American Stroke Association. Stroke 44, 2064-2089. https://doi.org/10.1161/STR.0b013e318296aeca (2013).

30. Li, H., Jia, J. \& Yang, Z. Mini-mental state examination in elderly Chinese: a population-based normative study. J. Alzheimers Dis. 53, 487-496 (2016).

31. Román, G. C. et al. Vascular dementia: diagnostic criteria for research studies. Neurology 43, 250-250 (1993).

32. Yang, J. et al. Risk factors for incident dementia after stroke and transient ischemic attack. Alzheimers Dement. 11, 16-23 (2015).

33. Fazekas, F., Chawluk, J. B., Alavi, A., Hurtig, H. I. \& Zimmerman, R. A. MR signal abnormalities at 1.5 T in Alzheimer's dementia and normal aging. Am. J. Roentgenol. 149, 351-356. https://doi.org/10.2214/ajr.149.2.351 (1987).

34. Xiong, Y. et al. Operational definitions improve reliability of the age-related white matter changes scale. Eur. J. Neurol. 18, 744-749. https://doi.org/10.1111/j.1468-1331.2010.03272.x (2011).

35. Inzitari, D. et al. Risk of rapid global functional decline in elderly patients with severe cerebral age-related white matter changes: the LADIS study. Arch. Intern. Med. 167, 81-88 (2007).

36. Ferguson, K. et al. Visual rating scales of white matter hyperintensities and atrophy: comparison of computed tomography and magnetic resonance imaging. J. Stroke Cerebrovasc. Dis. 27, 1-8. https://doi.org/10.1016/j.jstrokecerebrovasdis.2018.02.028 (2018).

37. Jin, H. et al. Prevalence and risk factors of white matter lesions in Tibetan patients without acute stroke. Stroke 51, 149-153. https:// doi.org/10.1161/STROKEAHA.119.027115 (2020).

38. O'donnell, M. J. et al. Risk factors for ischaemic and intracerebral haemorrhagic stroke in 22 countries (the INTERSTROKE study): a case-control study. Lancet 376, 112-123 (2010).

39. Sudre, C. H. et al. Cardiovascular risk factors and white matter hyperintensities: difference in susceptibility in South Asians compared with Europeans. J. Am. Heart Assoc. 7, e010533. https://doi.org/10.1161/JAHA.118.010533 (2018).

40. Ossenkoppele, R. et al. Prevalence of amyloid PET positivity in dementia syndromes: a meta-analysis. JAMA 313, 1939-1949 (2015).

41. Ylikoski, A. et al. White matter hyperintensities on MRI in the neurologically nondiseased elderly: analysis of cohorts of consecutive subjects aged 55 to 85 years living at home. Stroke 26, 1171-1177. https://doi.org/10.1161/01.str.26.7.1171 (1995).

42. de Leeuw, F. E. et al. Prevalence of cerebral white matter lesions in elderly people: a population based magnetic resonance imaging study. The Rotterdam Scan Study. J. Neurol. Neurosurg. Psychiatry 70, 9-14. https://doi.org/10.1136/jnnp.70.1.9 (2001).

43. Schmidt, R., Enzinger, C., Ropele, S., Schmidt, H. \& Fazekas, F. Progression of cerebral white matter lesions: 6-year results of the Austrian stroke prevention study. Lancet 361, 2046-2048. https://doi.org/10.1016/S0140-6736(03)13616-1 (2003).

44. van Dijk, E. J. et al. Progression of cerebral small vessel disease in relation to risk factors and cognitive consequences: Rotterdam Scan Study. Stroke 39, 2712-2719. https://doi.org/10.1161/strokeaha.107.513176 (2008).

45. Jimenez-Conde, J. et al. Hyperlipidemia and reduced white matter hyperintensity volume in patients with ischemic stroke. Stroke 41, 437-442. https://doi.org/10.1161/strokeaha.109.563502 (2010).

46. Longstreth, W. Jr. et al. Incidence, manifestations, and predictors of worsening white matter on serial cranial magnetic resonance imaging in the elderly: the Cardiovascular Health Study. Stroke 36, 56-61 (2005). 
47. De Bresser, J. et al. Progression of cerebral atrophy and white matter hyperintensities in patients with type 2 diabetes. Diabetes Care 33, 1309-1314 (2010).

48. Kooistra, M. et al. Diabetes mellitus and progression of vascular brain lesions and brain atrophy in patients with symptomatic atherosclerotic disease. The SMART-MR study. J. Neurol. Sci. 332, 69-74 (2013).

49. Van Elderen, S. et al. Progression of brain atrophy and cognitive decline in diabetes mellitus: a 3-year follow-up. Neurology 75, 997-1002 (2010).

50. Shim, Y. S., Yang, D. W., Kim, H.-J., Park, Y. H. \& Kim, S. Characteristic differences in the mini-mental state examination used in Asian countries. BMC Neurol. 17, 141-141. https://doi.org/10.1186/s12883-017-0925-z (2017).

51. Mok, V. C. et al. Delayed-onset dementia after stroke or transient ischemic attack. Alzheimers Dement. 12, 1167-1176 (2016)

52. van Middelaar, T. et al. Effect of antihypertensive medication on cerebral small vessel disease: a systematic review and meta-analysis. Stroke 49, 1531-1533 (2018)

53. Livingston, G. et al. Dementia prevention, intervention, and care: 2020 report of the Lancet Commission. The Lancet 396, $413-446$ (2020).

54. Nasrallah, I. M. et al. Association of intensive vs standard blood pressure control with cerebral white matter lesions. JAMA 322, 524-534 (2019).

55. Williamson, J. D. et al. Effect of intensive vs standard blood pressure control on probable dementia: a randomized clinical trial. JAMA 321, 553-561 (2019).

56. Ott, B. R. et al. Do statins impair cognition? A systematic review and meta-analysis of randomized controlled trials. J. Gen. Intern. Med. 30, 348-358. https://doi.org/10.1007/s11606-014-3115-3 (2015).

57. Zhang, H. et al. Effects of sartans and low-dose statins on cerebral white matter hyperintensities and cognitive function in older patients with hypertension: a randomized, double-blind and placebo-controlled clinical trial. Hypertens. Res. 42, 717-729 (2019).

\section{Acknowledgements}

This study was financially supported by the National Key Research and Development Program of China (Grant Number 2016YFC1300600), Health and Health Services Research Fund (0708041) of the Food and Health Bureau of the Government of HKSAR General Research Fund (Grant Number GRF CUHK 471911), the Lui Che Woo Institute of Innovative Medicine, and Therese Pei Fong Chow Research Centre for Prevention of Dementia (in memory of Donald H. K. Chow). We acknowledge Eugene S. K. Lo for the coordination and data collection of the project and Vincent Hui for the preparation of Supplementary Figure 1.

\section{Author contributions}

B.Y.K.L., A.Y.L.L. and V.C.T.M. designed and conceptualized study. B.Y.K.L., B.Y. and K.T.L., analyzed the data. B.Y.K.L. drafted the manuscript for intellectual content. B.Y., E.A., C.L.H.C., Y.D., J.C.D., P.V.G., S.H., N.K., S.Y.K, J.Y.L., P.A.O., V.S., K.T.L., H.W., Y.H.Y., T.Y., F.A., S.A., S.W., H.K., A.Y.L.L. and V.C.T.M. interpreted the data. B.Y., E.A., C.L.H.C., Y.D., J.C.D., P.V.G., S.H., N.K., S.Y.K, J.Y.L., P.A.O., V.S., H.W., Y.H.Y., T.Y., F.A., S.A., S.W., H.K., A.Y.L.L. and V.C.T.M. revised the manuscript for intellectual content. E.A., C.L.H.C., Y.D., J.C.D., P.V.G., S.H., N.K., S.Y.K, J.Y.L., P.A.O., V.S., H.W., Y.H.Y., F.A., S.A., and V.C.T.M. had a major role in data acquisition.

\section{Competing interests}

We acknowledge Otsuka Pharmaceutical Company Limited (Korea Otsuka International Asia Arab) for providing partial financial support in conducting the AWARE study. However, they had no input in the study design, interpretation of the results, or preparation of the manuscript. There is no competing interest of all authors.

\section{Additional information}

Supplementary information The online version contains supplementary material available at https://doi.org/ 10.1038/s41598-021-90746-x.

Correspondence and requests for materials should be addressed to A.Y.L.L.

Reprints and permissions information is available at www.nature.com/reprints.

Publisher's note Springer Nature remains neutral with regard to jurisdictional claims in published maps and institutional affiliations.

Open Access This article is licensed under a Creative Commons Attribution 4.0 International License, which permits use, sharing, adaptation, distribution and reproduction in any medium or format, as long as you give appropriate credit to the original author(s) and the source, provide a link to the Creative Commons licence, and indicate if changes were made. The images or other third party material in this article are included in the article's Creative Commons licence, unless indicated otherwise in a credit line to the material. If material is not included in the article's Creative Commons licence and your intended use is not permitted by statutory regulation or exceeds the permitted use, you will need to obtain permission directly from the copyright holder. To view a copy of this licence, visit http://creativecommons.org/licenses/by/4.0/.

(C) The Author(s) 2021 